\title{
Kernos
}

Revue internationale et pluridisciplinaire de religion grecque antique

$16 \mid 2003$

Varia

\section{A. MELE, M. TORTORELLI GHIDINI, Epimenide cretese}

\section{Marie-Christine Leclerc}

\section{(2) OpenEdition}

\section{Journals}

Édition électronique

URL : http://journals.openedition.org/kernos/842

DOI : $10.4000 /$ kernos. 842

ISSN : 2034-7871

Éditeur

Centre international d'étude de la religion grecque antique

Édition imprimée

Date de publication : 1 janvier 2003

Pagination : 367-368

ISSN : 0776-3824

Référence électronique

Marie-Christine Leclerc, «A. MELE, M. TORTORELLI GHIDINI, Epimenide cretese », Kernos [En ligne], 16 | 2003, mis en ligne le 14 avril 2011, consulté le 24 septembre 2020. URL : http://journals.openedition.org/ kernos/842 ; DOI : https://doi.org/10.4000/kernos.842 
A. Mele, M. Tortorelli Ghidini (éds), Epimenide cretese, Napoli, Luciano Editore, 2001. 1 vol. $15 \times 21 \mathrm{~cm}, 441$ p. (Quademi del Dipartimento di Discipline Storiche "Ettore Lepore". Università "Federico II", 2). ISBN : 88-88141-24-3.

Ce volume rassemble les contributions des treize participants à un séminaire sur Épiménide de Crète organisé par A. Mele et M. Tortorelli Ghidini le 3 décembre 1999 à l'Université Federico II de Naples (département cité en référence). Ce dossier, riche et bien documenté, dont une table de concordances et trois index rendent la consultation facile, présente une analyse des différents traits prêtés au sage crétois (le prophète, le purificateur, le refondateur, le « chamane »...) ainsi que des fragments d'une œuvre qui lui est attribuée.

L'ouvrage se divise en trois parties d'inégale longueur: La vita, regroupant les études de M. Gigante, P. Scarpi, J.C. Capriglione, M. Tortorelli Ghidini, E. Federico, A. Visconti et M. Lupi; L'opera (A. Bernabé, G. Arrighetti, A. Mele et L. Breglia Pulci Doria); La fortuna (M. Catarzi et G. Casertano). On s'attend donc à une démarche qui irait d'une tentative de reconstitution biographique aux traces laissées ultérieurement dans les esprits par les gestes et l'œuvre d'Épiménide, et aux interprétations auxquelles ceux-ci ont donné lieu. Or il n'en est rien. Seule la partie centrale, L'opera, correspond au titre qui la couronne. Celle-ci a une forte cohérence, dans la mesure où les quatre auteurs (cf. liste supra) explorent, souvent en détails et de manière systématique, les fragments d'une théogonie d'Épiménide dont ils reconstituent les principes cosmologiques. Au cours de cette investigation, la pensée du crétois est confrontée à celle d'auteur's notoires d'autres théogonies, et aussi à certaines théories des philosophes présocratiques, tant il est vrai qu'Épiménide pourrait se situer à la charnière entre tradition mythique et philosophie naissante.

À l'inverse, il n'est pas aisé de faire la part entre vita et fortuna, comme on peut s'y attendre s'agissant d'une tradition aussi indirecte que celle d'Épiménide. La première partie de l'ouvrage comporte, certes, des analyses historiques. C'est ainsi qu'E. Federico tente d'isoler un noyau de vérité concernant l'intervention d'Épiménide à Athènes : il a purifié la cité. Mais l'A. ajoute que la figure d'Épiménide a été récupérée à l'époque classique à des fins idéologiques. M. Lupi évoque la tradition spartiate, peu développée, d'un Épiménide principalement lié à la fonction prophétique. L'un des oracles qui s'impriment post mortem sur la peau du crétois serait à mettre en relation avec la tension entre roi et éphores à la fin du vi ${ }^{\mathrm{e}}$ siècle. Là aussi, il s'agit d'une utilisation intéressée. Nous ne sommes plus, dès lors, dans la logique d'une reconstitution historique au sens strict du terme. S'il s'agit bien de reconstituer des actes qui seraient historiquement attestés, il s'agit aussi, et peut-être surtout, d'explorer l'usage que telle cité fait d'une figure emblématique qu'elle utilise à des fins politiques. Ainsi, c'est davantage d'un Épiménide construit ou reconstruit que d'un Épiménide historique qu'il est question dans cette première partie : M. Gigante évalue le degré de crédibilité de la vie d'Épiménide selon Diogène Laërce; M. Tortorelli Ghidini récapitule les différents traits à partir desquels se constitue la figure du personnage.

S'ajoutent à ces investigations des questionnements plus anthropologiques, qui examinent les données concernant la vie d'Épiménide du point de vue du système de représentations grec. P. Scarpi présente le Crétois comme un être des confins, hantant les limites de l'humanité, circulant librement entre hommes et dieux, catégories pourtant séparées depuis le sacrifice prométhéen. Son altérité se manifeste, entre autres, par sa prescription d'un sacrifice humain à Athènes ou par son régime alimentaire atypique, commenté également par J.C. Capriglione. De ces analyses, il ressort qu'on se représente Épiménicle comme un marginal par rapport aux cadres dans lesquels la culture grecque pense l'humanité civilisée. Épiménide est (d')ailleurs; il agit dans cles cités qui ne sont pas la sienne. Cette distance lui est nécessaire pour ses œuvres prophétiques, purificatrices ou refondatrices destinées, elles, à rétablir l'ordre cles choses. Ceci n'a pas toujours été compris par les anciens. Ainsi, Épiménide a parfois été confondu avec Bouzygès, héros fondateur de l'agriculture, avec qui il n'a que des affinités fonctionnelles: il restaure une bonne agriculture après la période de crise à Athènes (A. Visconti). Au total, c'est déjà beaucoup plus de fortuna que de vita qu'il est question dans la première partie.

Aussi quand en fin d'ouvrage, dans la troisième partie, G. Casertano reprend quelques-uns de ces mêmes thèmes, ne peut-on se défendre d'une impression de redite; seules 
ses douze dernières pages concernant le paradoxe de menteur (qui valent davantage par leur documentation que par leur conceptualisation), entrent entièrement dans le thème de la fortuna. Enfin, l'étude de M. Catarzi sur le rôle du temps dans la légende d'Épiménide ajoute une dimension philosophique aux problénatiques précédentes. Elle montre que ce thème traverse toute la tradition concernant le sage crétois. Épiménicle pourrait être présenté comme le nom donné à un ensemble de figurations possibles du temps.

Marie-Christine Leclerc (Université de Toulouse-Le Mirail)

Marisa Tortorelli Ghidini, Alfredina Storchi Marino, Amedeo Visconti (éds), Tra Orfeo e Pitagora. Origini e incontri di culture nell'antichità. Atti dei seminari napoletani 1996-1998, Napoli, Bibliopolis, 2000. 1 vol. $17 \times 24$ cm, 575 p. ISBN : 887088-395-7.

Le projet scientifique interdisciplinaire dont ce volume rend compte avait pour thème «Origines et rencontres de cultures dans l'Antiquité » et comme aire d'enquête «L'Italie méridionale, entre Grecs et Romains »; il a conné lieu à trois séminaires internationaux dont on peut donc lire ici les Actes. Le volume comprend deux sections : l'une centrée sur l'orphisme, l'autre sur le pythagorisme, chacune renfermant onze essais, pour un total de vingt-deux textes d'un grand intérêt.

L'intérêt majeur me semble être l'approche concentrique des phénomènes pris en compte, dans la mesure où, par exemple pour la première partie, il est à la fois question des textes (A. Bernabé, Nuovi frammenti orfici e una nuova edizione degli 'Opфıka;) et de l'archéologie (A. Bottini, Forme di religiosilà salvifica in Magna Grecia. La documentazione arcbeologica); des Grecs, des Romains et des indigènes (P. Poccetti, La diffirione di dottrine misteriche e sapienziali nelle culture indigene dell'talia antica: appunit per un dossier); de philosophie (L. Brisson, Nascita di un mito filosofico: Giamblico (VP. 146) su Aglaophamos) et d'histoire des religions (D.M. Cosi, Orfeo e l'orfismo tra continuità e innovazione); de mythes et de rites; d'Orphée, mais aussi de Pythagore, de Dionysos et d'Apollon. Le phénomène est ainsi cerné de toutes parts et il en ressort une image extrêmement riche, problématique (dans le sens positif du terme), articulée. Mission remplie, donc, pour les promoteur's de ces séminaires si tant est, comme l'affirme M. Tortorelli Ghidini que "l'obiettivo del seminario di avviare un bilancio sul complesso di culti misterici e forme sapienziali che si manifestano nell'Italia centro-metidionale tra Greci e Romani consiglia di rinunziare a un disegno storico dell'orfismo, o meglio alle immagini che la moderna storiografia ne presenta ». Plutôt donc que de viser à une étude systématique, on procède ici par touches successives, en mettant l'accent sur les problèmes récents, les nouveaux textes, les questions à l'ordre du jour, en particulier la profonde solidarité qui unit orphisme, pythagorisme et dionysisme en Italie, comme ailleur's.

La seconde partie, centrée sur le pythagorisme, s'ouvre sur une contribution du regretté $G$. Giannantoni qui met remarquablement en avant les principaux enjeux du dossier : la conception doxographique des présocratiques, héritée de Diels, qui a tendance à gommer les différences et à créer une unité et une apparence d'évolution fallacieuses dans ce qui est en fait une multitude de courants culturellement très différenciés; la connaissance indirecte que nous avons de Pythagore et de son enseignement à la faveur du renouveau de son école, des siècles plus tard a pour conséquence la difficulté de faire le tri entre ce qui appartient au mâ̂tre, donc aux origines du mouvement, et ce qui relève des diadoques récents, Philolaos et Archytas en particulier, vus par des auteurs qui vivaient encore une ou plusieurs générations après. En outre, on n'oubliera pas que la secte pythgoricienne était vouée au silence, au secret, à l'isolement et au culte de son fondateur, ce qui rend particulièrement ardue la reconstruction de son enseignement doctrinaire originel. Enfin, au nombre des questions centrales du débat sur le pythagorisme, se trouve naturellement la valeur du témoignage aristotélicien. Témoignage précieux et informé selon les uns, superficiel et polémique selon les autres. Cette question s'avère importante pour formuler une évaluation de la nature même du pythagorisme: doctrine éthique et religieuse de purification ou doctrine philosophique et scientifique? Il n'est du 